\title{
Investigation of Solar/Heliospheric Anomalies Associated with the Solar Minimum of 2007 - 2008
}

\author{
Chin-Chun $\mathrm{Wu}^{1, *}, \mathrm{Kan} \mathrm{Liou}^{2}$, Simon Plunkett ${ }^{1}$, Craig Daniel Fry ${ }^{3}$, and Shi-Tsan $\mathrm{Wu}^{4}$ \\ ${ }^{1}$ Space Science Division, Naval Research Laboratory, Washington, DC, USA \\ ${ }^{2}$ Applied Physics Laboratory, Johns Hopkins University, Laurel, Maryland, USA \\ ${ }^{3}$ Exploration Physics International, Inc., Huntsville, Alabama, USA \\ ${ }^{4}$ Center for Space Plasma and Aeronomic Research (CSPAR), University of Alabama, Huntsville, Alabama, USA
}

Received 18 March 2012, accepted 16 October 2012

\begin{abstract}
The unusually long-extended solar minimum between cycles 23 and 24 (from 2007 to 2008) yielded a number of anomalies with regard to solar/heliospheric phenomena wherein the solar wind magnetic field is $36 \%$ weaker than that for the previous solar cycle minimum (from 1996 to 1997) at $1 \mathrm{AU}$, the solar wind dynamic pressure is the lowest observed since the beginning of the space age, the unusually high tilted angle of the solar dipole, and the absence of a classical quiescent equatorial streamer belt. To understand the cause of the anomalies, we perform numerical simulation of a realistic inner heliosphere using a global three-dimensional, time-dependent, numerical model with observed solar inputs. It is suggested that these solar extremes are associated with (1) an inflated heliospheric current/plasma sheet (HCS/HPS) and (2) a decrease in the integrated fluxes of mass and magnetic field ejected from the Sun, which was manipulated by some unknown internal solar dynamics.
\end{abstract}

Key words: Interplanetary magnetic field, Solar cycle, Heliospheric current sheet, Solar anomaly

Citation: Wu, C. C., K. Liou, S. Plunkett, C. D. Fry, and S. T. Wu, 2013: Investigation of solar/heliospheric anomalies associated with the solar minimum of 2007 - 2008. Terr. Atmos. Ocean. Sci., 24, 243-252, doi: 10.3319/TAO.2012.10.16.01(SEC)

\section{INTRODUCTON}

The heliospheric current sheet (HCS) (Wilcox and Ness 1965) is a thin current layer near the solar equator that borders the outward directed and the inward directed open heliospheric magnetic field lines. At higher latitudes, the HCS is wrapped by a layer of low magnetic field and velocity but high-density solar wind material which is called the heliospheric plasma sheet (HPS) (e.g., Borrini et al. 1981; Gosling et al. 1981; Burlaga et al. 1990). The typical shape of the HCS/HPS is flat during solar minimum periods and becomes highly warped and tilted during solar maximum periods (e.g., Badruddin et al. 2007; Hu et al. 2008). As a consequence, during solar minimum the typical value of solar wind density is high and the interplanetary magnetic fields (IMFs) are low in the ecliptic plane, as observed by the IMP-8, WIND, and/or ACE spacecraft (e.g., also see Fig. 1).

\footnotetext{
* Corresponding author

E-mail: chin-chun.wu@nrl.navy.mil
}

At $1 \mathrm{AU}$, the characteristics of the solar wind plasma and IMF are still, to a large extent, controlled by the Sun's activity. During solar minimum, the Sun's magnetic field is dipole-like, with helmet streamers associated with closed magnetic field near the equator, and with coronal holes associated with open magnetic field in the pole regions. Solar wind speed associated with the streamers is slow and the plasma is dense, but fast and tenuous at the coronal holes. During the solar minimum between cycles 23 and 24 (2007 - 2008), solar wind plasma and IMF data near the Earth's orbit indicate a different scenario. For example, the averaged solar wind magnetic field was lower than its value in the previous solar minimum (between cycles 22 and 23,1996$)$ and the solar wind global pressure was the lowest observed since the beginning of the space age (e.g., Luhmann et al. 2009). Observations of the HCS in the solar minimum of cycles 23 and 24 show that the HCS did not lie in the equatorial region but was inclined to the equatorial plane and extended to mid helio-latitudes (e.g., Wang et al. 2009). Because the HCS structure along the Earth's 
orbit is determined mainly by its properties near the solar surface; tracing the properties of the HCS and its surrounding plasma and field out to $1 \mathrm{AU}$ and compared with the in situ measurements can provide a reasonable explanation for these observations. The main objective of this study is to understand the causes of in situ plasma and magnetic field measurements at $1 \mathrm{AU}$ during the solar minimum of 2007 - 2008. To assist in the interpretation of observational

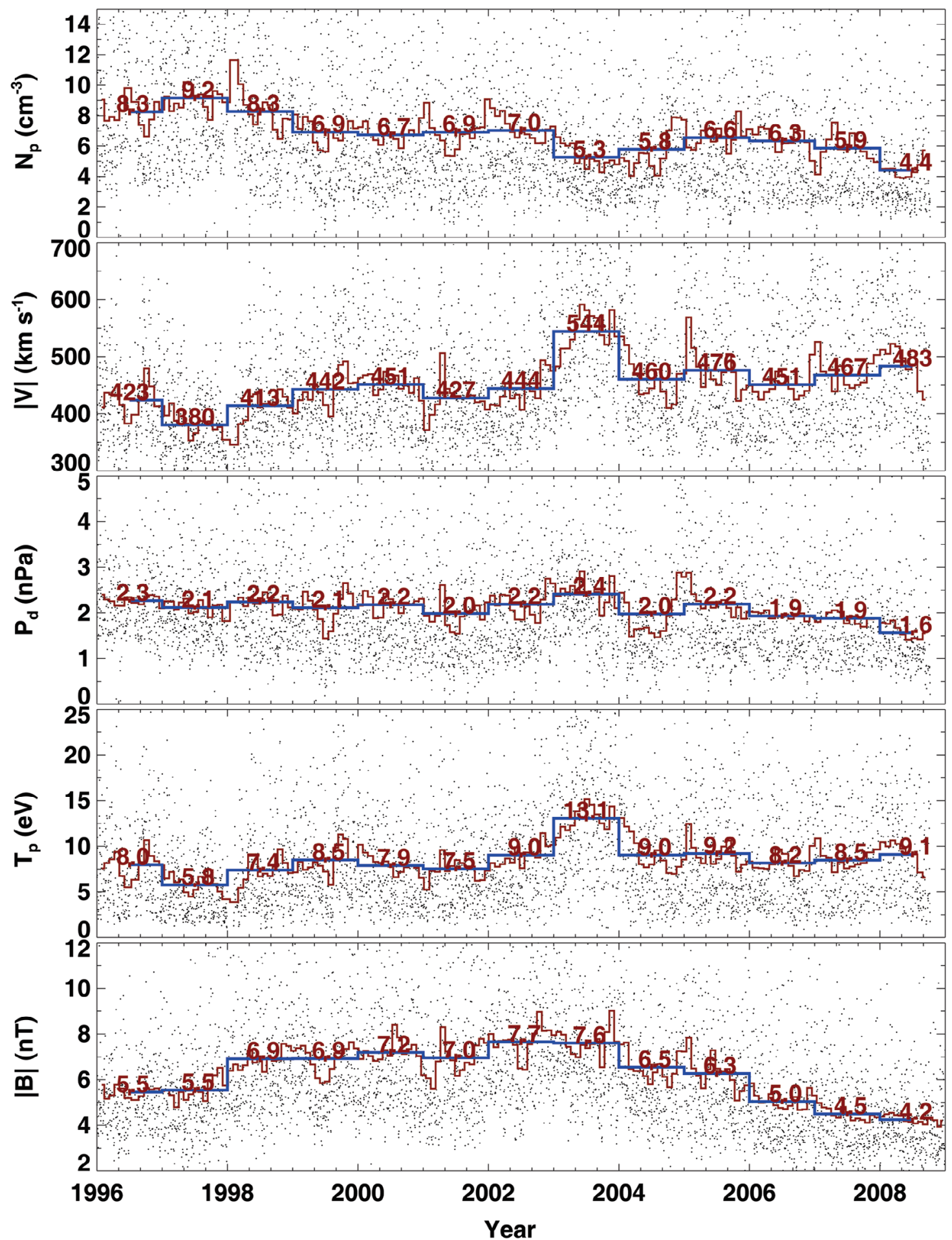

Fig. 1. Observations of solar wind proton density, velocity, dynamic pressure ( $\equiv 1.6726 \times 10^{-6} \mathrm{~N}_{\mathrm{p}} \mathrm{V}^{2}$, units of $\mathrm{N}_{\mathrm{p}}$ is $\mathrm{cm}^{-3}$, and $\mathrm{V}$ is km s $\left.\mathrm{s}^{-1}\right)$, temperature, and magnetic fields (from top to bottom) between the previous solar minimum (1996 - 1997) and the current solar minimum (2007 - 2008). The black dots represent daily averages, the solid blue curves represent 27 days' running averages. The red solid lines represent yearly averages, which are also shown on the top of the red lines (WIND data were used for the period 1996 - 1998; ACE data were used for the period 1999 - 2008). 
results, a validated, state-of-art global three-dimensional (3-D) time-dependent, numerical simulation model, driven by actual solar inputs is used to simulate the realistic heliosphere, including the 3-D structure of the HCS/HPS. Solar wind plasma and magnetic field observations from 1995 to 2008 are presented in section 2 . In section 3 , we propose a "HCS/HPS inflation hypothesis" to explain why both solar wind density and IMF were extremely low during the solar minimum of cycle $23 / 24$, followed by a summary of Conclusions in sections 4 .

\section{IN SITU MEASUREMENTS AT 1 AU}

Figure 1 shows L1 in situ observations of solar wind proton density, velocity, dynamic pressure $\left(P_{d}\right.$ in $\mathrm{nPa}=$ $1.6726 \times 10^{-6} \mathrm{~N}_{\mathrm{P}} \mathrm{V}^{2}$, units of $\mathrm{N}_{\mathrm{P}}$ is $\mathrm{cm}^{-3}$, and $\mathrm{V}$ is $\mathrm{km} \mathrm{s}^{-1}$ ), temperature, and magnetic field (from top to bottom) between the previous solar minimum (over the period of 1996 - 1997: between solar cycles 22 and 23) and the current solar minimum (over the period of 2007 - 2008: between solar cycles 23 and 24). The black dots represent daily averages and the solid red curves represent 27-day running averages. Since ACE started collecting L1 solar wind data in 1999 and WIND was occasionally orbiting in the magnetosphere after 1999, WIND data was used for the period 1996 - 1998; ACE data was used for the period 1999 - 2008 in this study.

The Sun has been less active during this solar minimum between solar cycle 23/24 than at any time since the 1970s. A number of "lows" in solar and heliospheric parameters have been observed from in situ solar wind data. For example, the following phenomena can be seen in Fig. 1 . (1) The solar wind magnetic field is $36 \%$ weaker than during the minimum of the previous solar cycle. (2) The lowest solar wind global pressure has been observed since the beginning of the space age. (3) The solar wind dynamic pressure is extremely low $(\sim 30 \%$ lower than the previous solar minimum). And, (4) the solar wind bulk speed is higher than the previous solar minimum. Both the solar wind speed and proton density contribute to solar wind dynamic pressure. Since solar wind speed is higher in the current solar minimum, it is clear that the extremely low dynamic pressure is caused by the low solar wind proton density (see the top three panels of Fig. 1).

The typical HCS/HPS has a low tilt angle with respect to the solar equator during the solar minimum period. In the region of the HCS/HPS, the solar wind speed and magnetic field are low but the solar wind density is high. For example, Fig. 2 is a schematic showing the three components of the solar wind at sunspot minimum (adapted from Fig. 12 of Wang et al. 2000). However, this paradigm does not hold for the current solar minimum for the period 2007 - 2008. A new hypothesis is needed to explain why the paradigm does not hold.

\section{NUMERICAL SIMULATION}

This study employs a global 3-D, time-dependent, numerical simulation magnetohydrodynamic (MHD) model with the aid of the Hakamada-Akasofu-Fry version 2, (HAFv.2) model (Hakamada and Akasofu 1982; Fry et al. 2001; Wu et al. 2007a, b) to simulate the structure of heliosphere within $1 \mathrm{AU}$. The numerical simulation code uses measured synoptic photospheric line-of-sight magnetograms that are extrapolated to $2.5 \mathrm{R}_{\mathrm{s}}$ by the Wang-Sheeley-Arge (WSA) model (Wang and Sheeley 1990; Wang et al. 1990; Arge and Pizzo 2000) as the boundary conditions at the surface of the Sun. The model used in this study is the composite of HAFv.2 model (see Fry et al. 2001, and references therein) and a fully 3-D, time-dependent MHD simulation code (Han et al. 1988; Detman et al. 1991, 2006). The HAFv. 2 code is a physics-based kinematic model which uses a modified kinematic approach to simulate the solar wind conditions out to $18 \mathrm{R}_{\mathrm{s}}$ with input data from Carrington Rotation maps $\left(2.5 \mathrm{R}_{\mathrm{s}}\right)$, provided by NOAA's Space Environment Center (http://swpc.noaa.gov). The WSA model results are evolved outward from 2.5 to $18 \mathrm{R}_{\mathrm{s}}$ by the HAFv. 2 model. HAFv. 2 computes the interaction of high-low speed streams that leads to the compression and rarefaction of plasma and magnetic field in this region. The outputs of HAFv. 2 at $18 \mathrm{R}_{\mathrm{s}}(0.08 \mathrm{AU})$ provide inputs for the time-dependent 3-D MHD solar wind model. The system is driven by a time series of synoptic photospheric magnetic maps composed from daily solar photospheric magnetograms (http://wso.stanford.edu). Use of these data

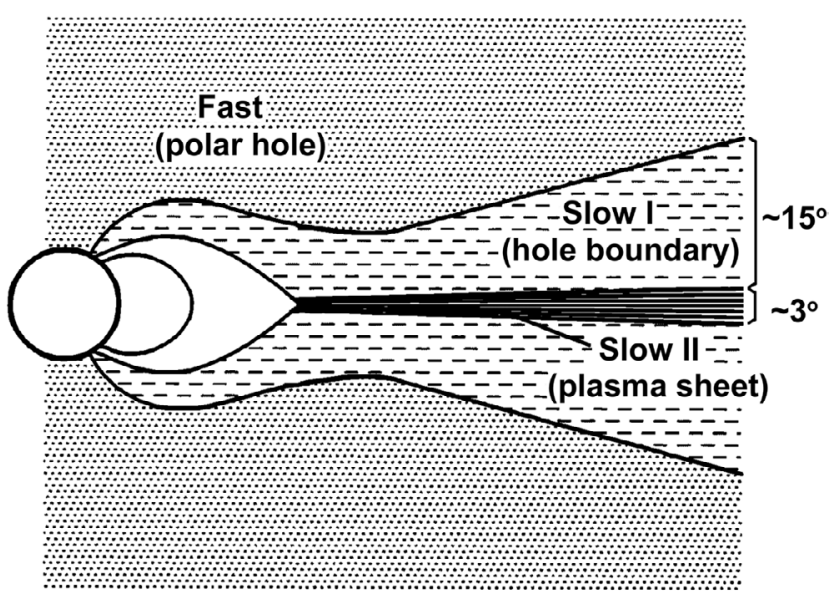

Fig. 2. Schematic showing the three components of the solar wind at sunspot minimum. High-speed wind escapes along the slowly diverging, open field lines in the polar hole interiors. Component I of the slow wind flows out along the rapidly diverging, open field lines rooted just inside the polar hole boundaries, which are located near latitude $60^{\circ}$ in each solar hemisphere. Component II of the slow wind, which originates from the closed field zone equatorially-ward of the polar hole boundaries, is confined to the dense equatorial plasma sheet that extends beyond $r \sim 3 \mathrm{R}_{\mathrm{s}}$ [adapted from Wang et al. (2000)]. 
to provide solar wind velocity and radial IMF at $2.5 \mathrm{R}_{\mathrm{s}}$ is described by Arge and Pizzo (2000).

The numerical 3-D MHD scheme used in this analysis is an extension of the two-step Lax-Wendroff finite difference method (Lax and Wendroff 1960). When using the two-step Lax-Wendroff finite difference method, the governing equations are required to be written in conservation form. A complete description of this conversion can be found in the work by Han (1977). The governing equations, for an ideal, single fluid, perfect gas, include conservation of mass, equation of motion, conservation of energy, and induction equation. The model solves the basic conservation (mass, momentum, and energy) laws with the induction equation to account for nonlinear interaction between the flow and magnetic fields. These MHD governing equations are casted in a uniform spherical grid, and the model solves the governing equations in that grid. The computa- tional model solves the basic MHD governing equations in uniform, spherical grids. The computational domain for the 3-D MHD simulation is a sun-centered spherical coordinate system $(r, \theta, \varphi)$ oriented on the ecliptic plane. Earth is located at $r=215 \mathrm{R}_{\mathrm{s}}, \theta=0^{\circ}$, and $\varphi=0^{\circ}$. The domain covers $-87.5^{\circ} \leq \theta \leq 87.5^{\circ} ; 0^{\circ} \leq \varphi \leq 360^{\circ} ; 18 \mathrm{R}_{\mathrm{s}} \leq r \leq 285 \mathrm{R}_{\mathrm{s}}$. An open boundary condition at both $\theta=87.5^{\circ}$ and $\theta=-87.5^{\circ}$ is used so there are no reflective disturbances. A constant grid size of $\Delta r=3 \mathrm{R}_{\mathrm{s}}, \Delta \theta=5^{\circ}$, and $\Delta \varphi=5^{\circ}$ is used which results with $90 \times 36 \times 72$ grid points.

To investigate differences in the configuration of the solar wind between the solar minimum of solar cycle 22/23 and the solar minimum of solar cycle $23 / 24$, we select two time periods which the HCS tilted angle is flatter than other periods; one in 1997 and another in 2008. Figure 3 shows 3-D simulation results in the form of a Carrington rotation map of solar wind parameters at $18 \mathrm{R}_{\mathrm{s}}(\approx 0.1 \mathrm{AU})$ for two
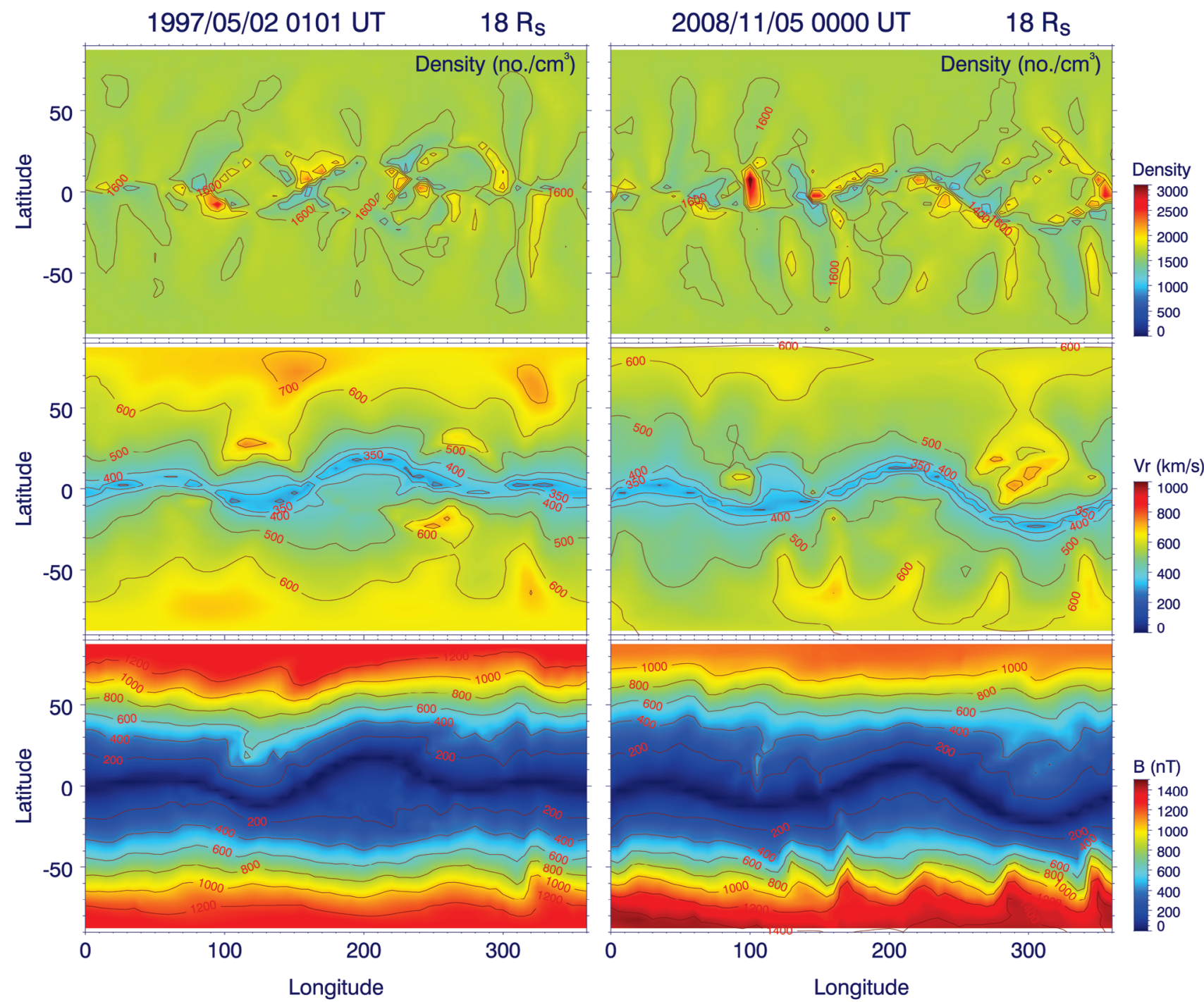

Fig. 3. Numerical simulations of density, velocity, and magnetic field profiles at 18 solar radii. Simulations are on 2 May 1997 (in the minimum of solar cycle 22/23), and 5 November 2008 (in the minimum of solar cycle 23/24) for the panels from left to right. 
different time periods. The top and bottom panels show the distribution of solar wind density, velocity, and magnetic fields, respectively. The left panel is a simulation of the background solar wind at $18 \mathrm{R}_{\mathrm{s}}$ representing the minimum of solar cycle $22 / 23$. The HCS/HPS is flat, as expected, and is located within $\pm 10^{\circ}$ of the ecliptic plane. The right panel of Fig. 3 shows the simulation result of the background solar wind in November 2008, which represents the minimum of solar cycle $23 / 24$. The HCS/HPS has a tilted angle ( $\sim$ with an omega shape and extends up to ecliptic latitudes of $\left.30^{\circ}\right)$.

We plotted the simulated background solar wind density and radial velocity at $215 \mathrm{R}_{\mathrm{s}}(\sim 1 \mathrm{AU})$ in Fig. 4 in the form of Carrington rotation maps. Comparing panels on the left (the 1997 event) and on the right (the 2008 event); the solar wind speed during the minimum of solar cycle $22 / 23$ is slower than it was during the minimum of solar cycle 23/24 in the solar equatorial region.
In general, the solar wind density is high, but both the velocity and magnetic field are low in the HCS (e.g., Eselevich and Filippov 1988; Crooker et al. 2004). However, Eselevich and Filippov (1988) also pointed out that the solar wind density is much lower in the region after the spacecraft passed through the HCS. Our numerical simulation results show similar phenomena. In both Figs. 3 and 4, there are some low-density regions near the HCS. For example, the blue band near $0^{\circ}$ ecliptic latitude represents a lower density region which is located within the high density areas (see red and bright yellow spots/bands in the upper panels of Fig. 3).

To provide more quantitative assessment of the differences in solar wind density and IMF associated with the two solar minimum times, we integrate fluxes of density and magnetic field for the two simulated periods at $215 \mathrm{R}_{\mathrm{s}}$ over three latitude bands: $47.5^{\circ}-87.5^{\circ}$ (high latitudes), $42.5^{\circ} \mathrm{N}$ -
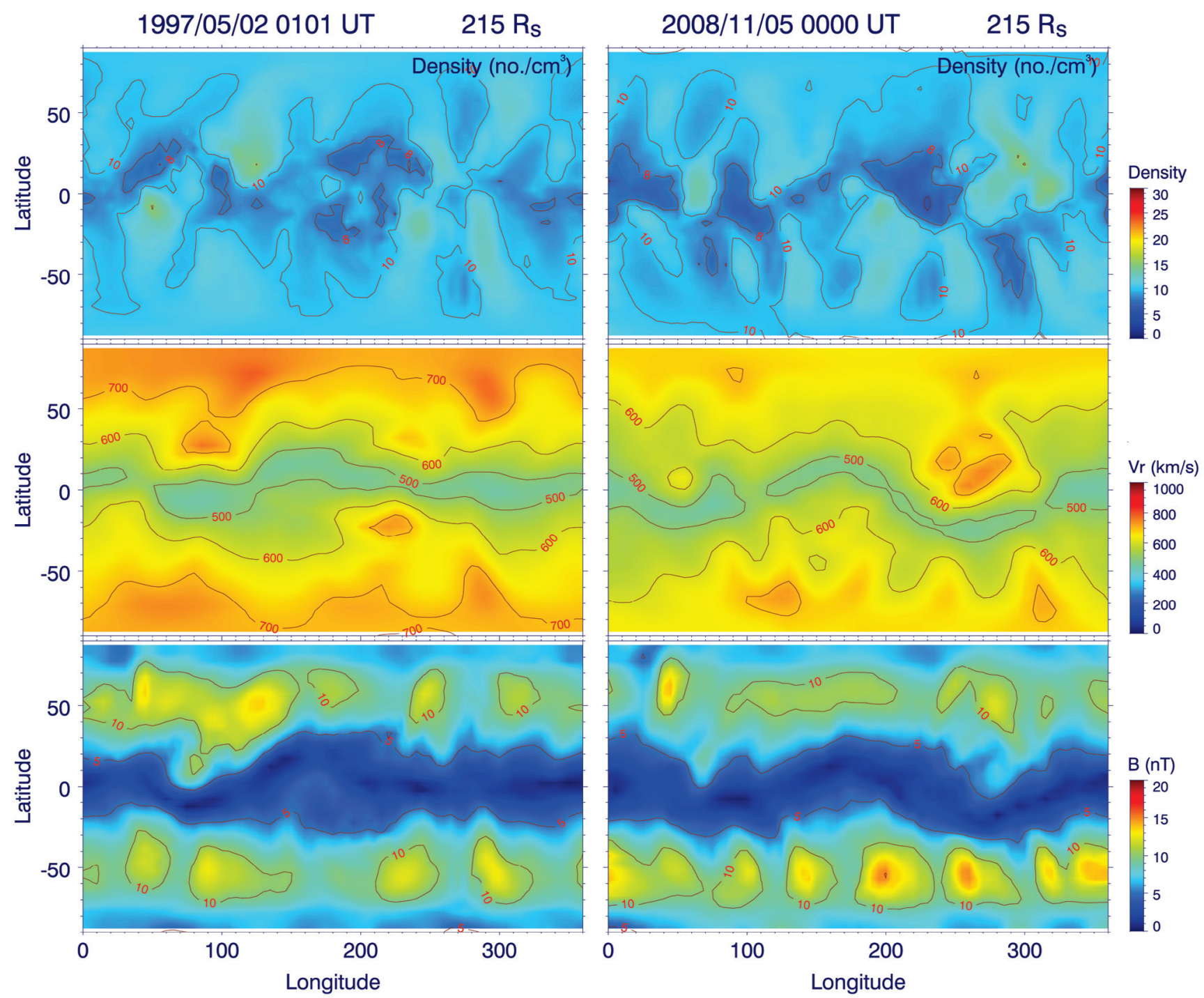

Fig. 4. Numerical simulations of density, velocity, and magnetic field profiles at 215 solar radii ( 1 AU). Simulations are on 2 May 1997 (in the minimum of solar cycle 22/23), and 5 November (in the minimum of solar cycle 23/24) for the panels from left to right. 
$42.5^{\circ} \mathrm{S}$ (mid-low latitudes), $17.5^{\circ} \mathrm{N}-17.5^{\circ} \mathrm{S}$ (low latitudes), and $87.5^{\circ} \mathrm{N}-87.5^{\circ} \mathrm{S}$ (global) centered on the Sun-Earth line. In Fig. 5, the left and right panels show the mass and IMF fluxes, respectively. The red-solid and blue-dotted lines represent the fluxes for the periods of May 1997 and November 2008, respectively. The density flux is higher in the solar equatorial region than in higher latitude areas (see Figs. 5a, $\mathrm{c}, \mathrm{e}, \mathrm{g})$. In contrast, the IMF flux is small near the solar equatorial plane and increases with latitudes (see Figs. 5b, $\mathrm{d}, \mathrm{f}, \mathrm{h}$ ). Simulation results show that there is a difference between the solar wind density and IMF fluxes for the two time periods, namely that the density and IMF fluxes were higher in May 1997 than in November 2008 (all red lines are higher than the blue dotted lines).

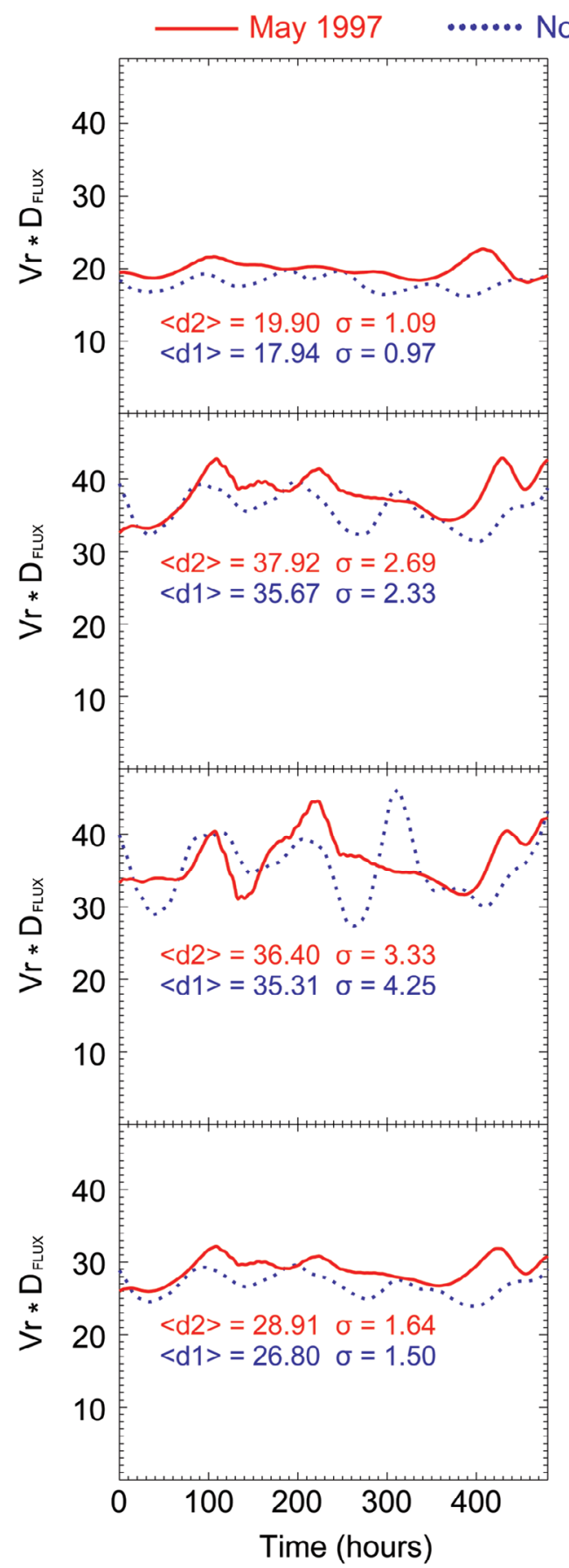

November 2008

Flux at $215 R_{s}$

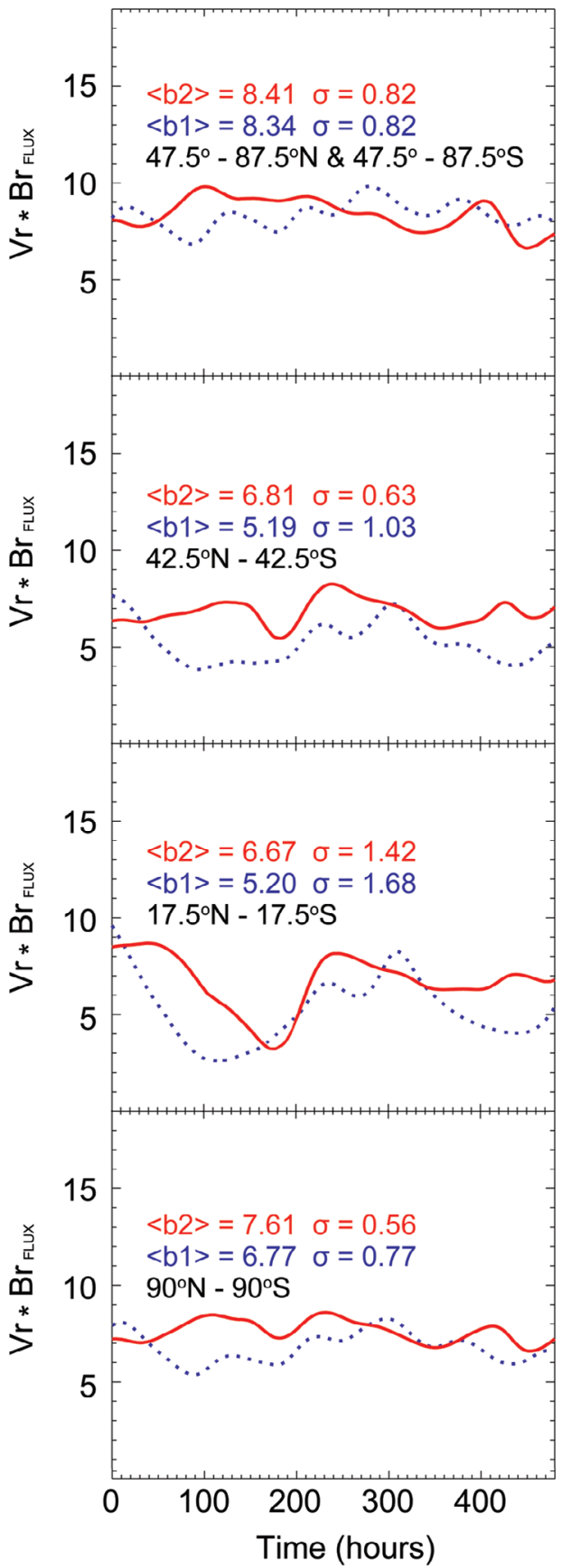

Fig. 5. Various fluxes for both solar wind (hourly resolution) density and interplanetary magnetic fields for the various areas. (a) (b): $47.5^{\circ}-87.5^{\circ}$; (c) $(\mathrm{d}): 42.5^{\circ} \mathrm{N}-42.5^{\circ} \mathrm{S}$; (e) (f): $17.5^{\circ} \mathrm{N}-17.5^{\circ} \mathrm{S}$; and (g) (h): $87.5^{\circ} \mathrm{N}-87.5^{\circ} \mathrm{S}$. Red-solid and Blue-dashed lines represent periods for May 1997 and November 2008. Note: $\sigma$ is standard deviation; $<\mathrm{d} 1>$ and $<\mathrm{d} 2>$ are the averages of density fluxes for the periods in November 2008 and May 1997 ; and $<\mathrm{b} 1>,<\mathrm{b} 2>$ are the averages of magnetic fluxes for the periods in November 2008 and May 1997. 


\section{DISCUSSION}

One of the key findings from our 3D MHD simulation is that both density and IMF fluxes near the solar equatorial plane in May 1997 were higher than those in November 2008. This is consistent with in situ measurements made by Wind and ACE (as shown in Fig. 1), which acquired solar wind and IMF data near the solar ecliptic plane. The present study result also shows that both density and IMF fluxes at lower latitudes $\left(\lambda<42.5^{\circ}\right)$ are also higher in 1997 than in 2008. In polar region, the magnetic flux in 1997 is higher than that in 2008. The result suggests a more inflated HCS/ HPS in 2008 than in 1997.

The lower density and IMF fluxes in 2008 could be one of the causes of the extremely low density and IMF observed at $1 \mathrm{AU}$. Our simulation result suggests that the solar coronal fields can expand further away from the solar equator because the IMF was weaker in November 2008 within $42.5^{\circ}$ from the solar equator (see Fig. 5d). This probably caused the small density flux in this period. It supports our HCS/HPS inflation hypothesis. In addition, our results also show that the global density flux in May 1997 is higher than in November 2008 (see Fig. 5e). Global density flux in May 1997 is only $7.3 \%$ higher than it was in November 2008. Differences of density flux for these two periods are $3.0 \%$, $5.9 \%$, and $9.9 \%$ for the lower, low-mid, and higher latitudes, respectively. Of course, simulations of more events that cover a longer period (e.g., the whole solar minima of cycle $22 / 23$ and 23/24) are necessary to confirm this point of view. We will address this in a future work.

The low solar magnetic field puts less pressure on the HCS/HPS, resulting in the inflation of the HCS/HPS away from the ecliptic plane. As a result, the HCS/HPS becomes thicker than it would otherwise appear. Assuming that the total solar wind mass flux is conserved, the solar wind density should be reduced in the region of inflated HCS/HPS. The conservation of flux also applies to the magnetic field. The inflation of the HCS/HPS causes the low solar wind density and magnetic field observed by the ACE/WIND spacecraft because mass flux should be constant in the HPS region. Figure 6 shows a schematic map of the HCS/HPS during solar minimum with different strengths of solar highlatitude magnetic fields. Dotted lines represent the location of the HCS which is wrapped by a thin layer of HPS. The Sun's high-latitude magnetic fields are weaker in the top panel of Fig. 6 than in the bottom panel of Fig. 6. Thus, an inflated HCS/HPS hypothesis may explain the unusual solar wind conditions observed during the solar minimum of cycle $23 / 24$. This is also supported by solar observations; magnetic fields in the high-latitude regions are weaker in the minimum of cycle 23/24 than normal (e.g., McComas et al. 2008; Wang and Sheeley 2009).

There are two possibilities for the low solar wind density during the solar minimum of cycle $23 / 24$; (1) lesser quantities of mass and magnetic field are ejected from the Sun than during the previous cycle, or (2) the inflation of HCS/HPS occurs as is discussed above. Figure 5 demonstrates that the total flux of magnetic fields is higher in May 1997 in both lower and higher latitude areas (see Figs. 5b, $\mathrm{d}, \mathrm{f}$ ). The averaged density in May 1997 is 7\% higher than that in November 2008. In a recent numerical simulation study by Yang et al. (2011), they have demonstrated that the weak polar fields in the minimum of cycle $23 / 24$ play an important role for solar wind anomalies which is consistent with the present results.

Bavassano et al. (1997) suggested that "the coronal counterpart of the plasma sheet is the stalk of the coronal streamer." The radial extension of the boundaries of the streamer is responsible for the observed brightness halo within a range of $15-20^{\circ}$ of the coronal streamer (see Fig. 7 ,

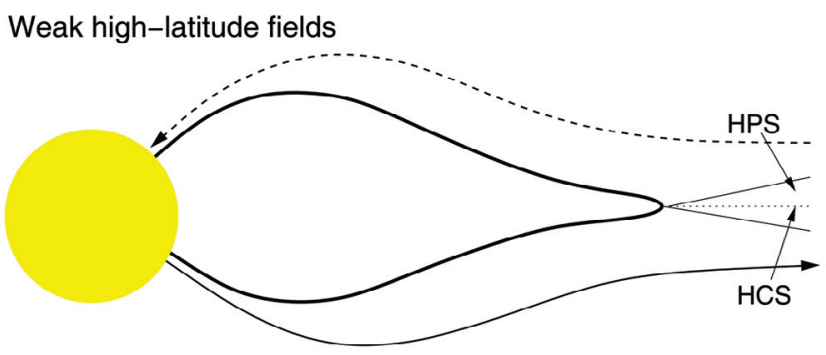

Strong high-latitude fields

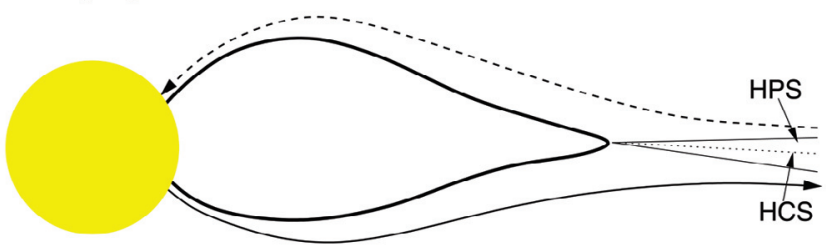

Fig. 6. Schematic showing the shape of HCS/HPS with different strengths of solar polar fields of the solar wind at solar minimum.

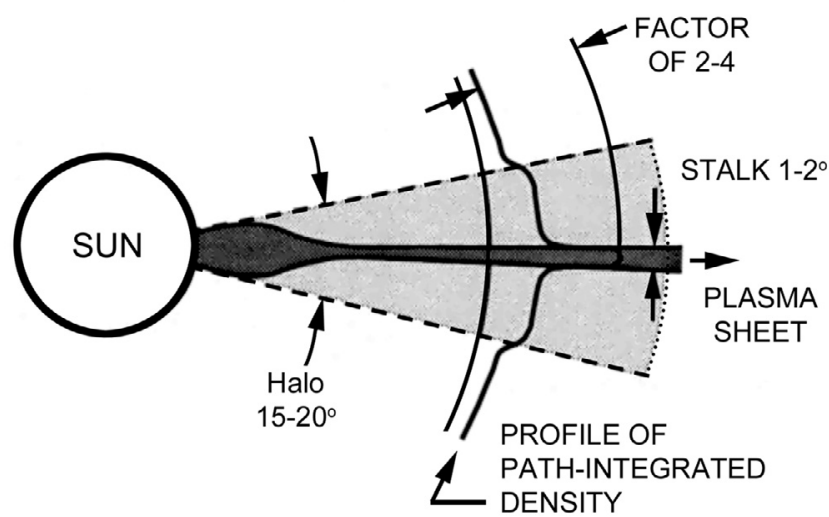

Fig. 7. An idealized coronal streamer and its stalk, which forms the plasma sheet in interplanetary space. The radial extension of the boundaries of the streamer is responsible for the observed density halo. The profile of path-integrated density is also shown (adapted from Bavassano et al. 1997). 
which is adapted from Fig. 5 of Bavassano et al. 1997). This brightness halo, corresponding to the integrated lineof-sight density, represents a "density halo." In Fig. 5, the choice of $\pm 17.5^{\circ}$ and $\pm 42.5^{\circ}$ for integration seems reasonable for covering the entire area of the "density halo." The integrated mass flux for the solar minimum of cycle 23/24 is less than the previous solar minimum (cycle 22/23), this supports the hypothesis that the inflation of the HCS/HPS is one factor causing the unusually low solar wind density and magnetic fields observed at $1 \mathrm{AU}$ during this solar minimum of cycle $23 / 24$.
In order to verify the hypothesis of the HCS/HPS inflation discussed above, we checked magnetic fields at $2.5 \mathrm{R}_{\mathrm{s}}$. Figure 8 shows the averages of magnetic fields at $2.5 R_{s}$ in different latitude regions. Table 1 summarizes the averages of magnetic fields in different regions (latitude) at $2.5 \mathrm{R}_{\mathrm{s}}$. Magnetic field in lower latitude (within $\pm 17.5^{\circ}$ ) in 1997 is weaker than that is in 2008 . In the regions within $\pm 42.5^{\circ}$ and higher latitude $\left(>42.5^{\circ}\right)$ magnetic fields are stronger in 1997 than that are in 2008. The hypothesis of HCS/HPS inflation (see Fig. 6) suggests that polar field may dominate the inflation of HCS/HPS. The higher latitude magnetic
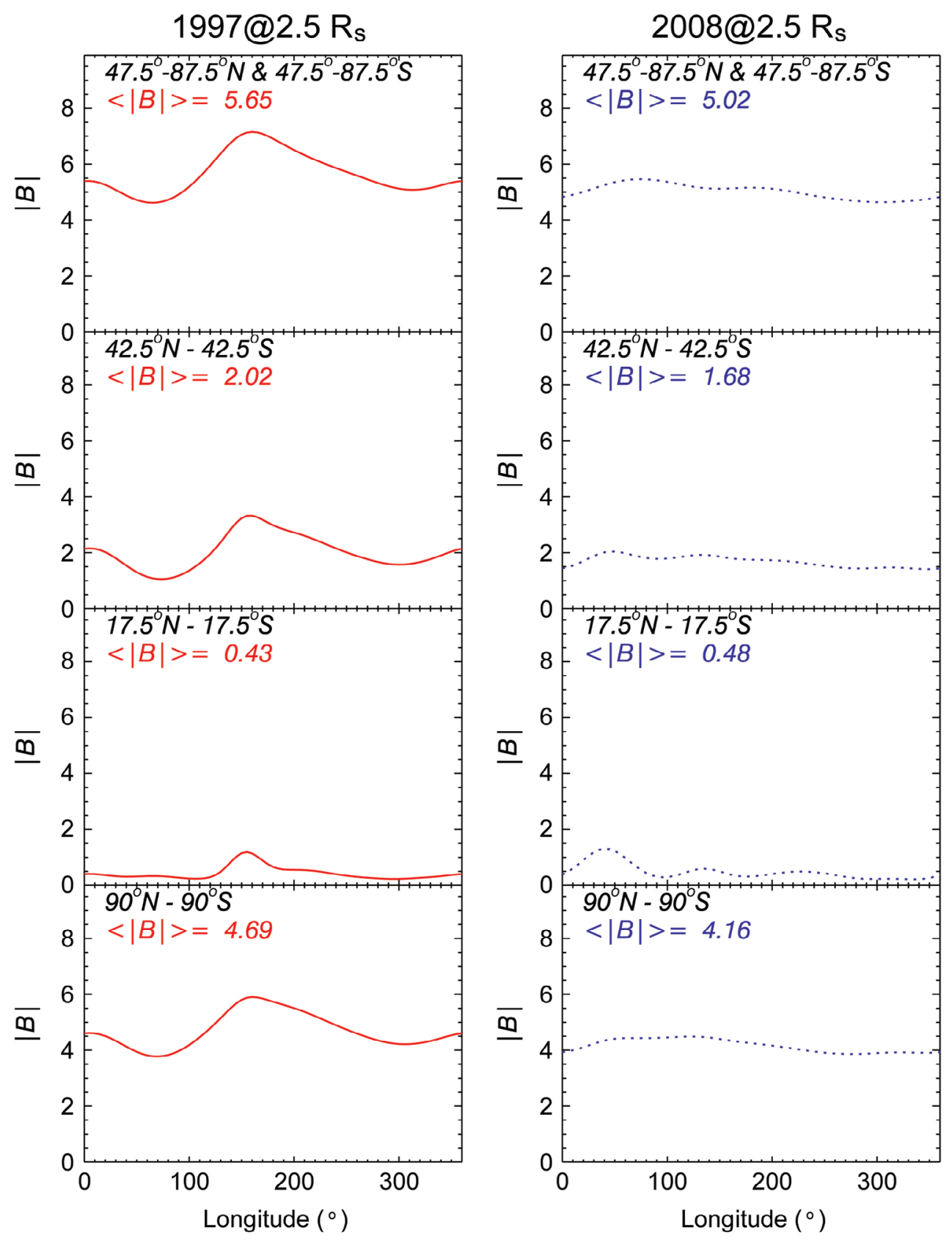

Fig. 8. Averaged magnetic fields at 2.5 solar radii for the various areas. From top to bottom: $47.5^{\circ}-87.5^{\circ} ; 42.5^{\circ} \mathrm{N}-42.5^{\circ} \mathrm{S} ; 17.5^{\circ} \mathrm{N}-17.5^{\circ} \mathrm{S}$; and $87.5^{\circ} \mathrm{N}-87.5^{\circ} \mathrm{S}$. Red-solid and Blue-dashed lines represent periods for May 1997, and November 2008. Unit of $|B|$ is in microtesla. 
Table 1. Averages of magnetic fields in different latitude regions at 2.5 solar radii.

\begin{tabular}{lcc}
\hline & May 1997 & November 2008 \\
\hline Region & $<B>$ (microtesla) & $<B>$ (microtesla) \\
$17.5^{\circ} \mathrm{S}^{\mathrm{a}} \leq$ Latitude $\leq 17.5^{\circ} \mathrm{N}^{\mathrm{b}}$ & 0.43 & 0.48 \\
$42.5^{\circ} \mathrm{S} \leq$ Latitude $\leq 42.5^{\circ} \mathrm{N}$ & 2.02 & 1.68 \\
Latitude $>42.5^{\circ}$ & 5.65 & 5.02 \\
$87.5^{\circ} \mathrm{S} \leq$ Latitude $\leq 87.5^{\circ} \mathrm{N}$ & 4.69 & 4.16 \\
\hline
\end{tabular}

Note: $a: S$ - South; $b: N$ - North.

field in 1997 is $~ 10 \%$ stronger than that in 2008 , but the lower latitude magnetic in 1997 is $\sim 10 \%$ weaker than that in 2008. In 2008, the stronger field at lower latitudes and the weaker polar field at $1 \mathrm{R}_{\mathrm{s}}$ caused the focus points of the open field regions and magnetic flux to be distributed more poleward at $2.5 \mathrm{R}_{\mathrm{s}}$ than in 1997. This had the effect of expanding the HCS/HPS further out to the higher latitudes in 2008 than in 1997. The stronger lower latitude magnetic field in 2008 also helps explain the expansion of HCS/HPS. Near the Sun, the magnetic field in low latitudes in 2008 is higher than in 1997 but the magnetic field at 1 AU near the ecliptic plane in 2008 is weaker than in 1997. The results of WSA's magnetostatic extrapolation of the line-of-sight magnetic field from 1 to $2.5 \mathrm{R}_{\mathrm{s}}$, driving HAFv.2's modified-kinematic evolution of the plasma to $18 \mathrm{R}_{\mathrm{s}}$, followed by the full 3D-MHD model's solution to the solar wind, are consistent with spacecraft observations at L1. Our proposed hypothesis of HCS/HPS inflation can explain why the magnetic field near the solar ecliptic plane at $1 \mathrm{AU}$ is weaker in the minimum of solar cycle 23/24 than in cycle 22/23.

\section{CONCLUSIONS}

In this study, we have used a numerical simulation to support our suggested causes for the solar/heliospheric anomalies observed during solar minimum for the cycle $23 / 24$, over the period of $2007-2008$. From the results presented in Fig. 5, it is clearly indicated that the amount of mass and magnetic flux ejected from the Sun into the heliosphere are smaller in comparison with cycle $22 / 23$. The current study is limited to near the equatorial region. This is because of the orbital restriction of the spacecraft (e.g., WIND, ACE). In fact, most in situ solar wind observations are collected near the solar ecliptic plane. It is difficult, if not possible, to investigate the causes of anomalies in the solar minimum of cycle $23 / 24$ using currently available data from these observations. As demonstrated in this study, global simulations can provide a good alternative in the absence of in situ observations for this type of work. We could conclude that less mass/magnetic field ejected from the Sun and the inflation hypothesis of the HCS/HPS are the two factors causing the unusually low solar wind density and magnetic field observed at $1 \mathrm{AU}$ (near the solar ecliptic plane) during the solar minimum of cycle 23/24.

Acknowledgements We thank the Wind PI team and National Space Science Data Center at Goddard Space Flight Center for management and providing Wind plasma and magnetic field solar wind data. This study is supported partially by NASA's LWS program via grants NNH09AM46I (CCW), NRL ISES and 6.1 programs (CCW and SP). STW is supported by NSF AGS1153323, and NASA/EPSCor-2009, NNx09AP74A Grant. Craig Daniel Fry's participation is supported by University of Alabama Huntsville Sub-Award SUB2010-045 under NASA Grant NNX09AP74A. Kan Liou is supported by NSF Grant. The Hakamada-AkasofuFry solar wind model version 2 (HAFv2) was provided to NRL/SSD by a software license from Exploration Physics International, Inc. (EXPI).

\section{REFERENCES}

Arge, C. N. and V. J. Pizzo, 2000: Improvement in the prediction of solar wind conditions using near-real time solar magnetic field updates. J. Geophys. Res., 105, 10465-10479, doi: 10.1029/1999JA000262. [Link]

Badruddin, M. Sigh, and Y. P. Sigh, 2007: Modulation loops, time lag and relationship between cosmic ray intensity and tilt of the heliospheric current sheet. Astron. Astrophys., 466, 697-704, doi: 10.1051/0004-6361:20 066549. [Link]

Bavassano, B., R. Woo, and R. Bruno, 1997: Heliospheric plasma sheet and coronal streamers. Geophys. Res. Lett., 24, 1655-1658, doi: 10.1029/97GL01630. [Link]

Borrini, G., J. T. Gosling, S. J. Bame, W. C. Feldman, and J. M. Wilcox, 1981: Solar wind helium and hydrogen structure near the heliospheric current sheet: A signal of coronal streamers at 1 AU. J. Geophys. Res., 86, 4565-4573, doi: 10.1029/JA086iA06p04565. [Link]

Burlaga, L. F., W. H. Mish, and Y.C. Whang, 1990: Coalescence of recurrent streams of different sizes and amplitudes. J. Geophys. Res., 95, 4247-4255, doi: 10.1029/ JA095iA04p04247. [Link]

Crooker, N. U., C. L. Huang, S. M. Lamassa, D. E. Larson, S. W. Kahler, and H. E. Spence, 2004: Heliospheric plasma sheets. J. Geophys. Res., 109, A03107, doi: 10. 1029/2003JA010170. [Link]

Detman, T. R., M. Dryer, T. Yeh, S. M. Han, S. T. Wu, and D. J. McComas, 1991: A time-dependent, three-dimensional MHD numerical study of interplanetary magnetic draping around plasmoids in the solar wind. J. Geophys. Res., 96, 9531-9540, doi: 10.1029/91JA00443. [Link]

Detman, T., Z. Smith, M. Dryer, C. D. Fry, C. N. Arge, 
and V. Pizzo, 2006: A hybrid heliospheric modeling system: Background solar wind. J. Geophys. Res., 111, A07102, doi: 10.1029/2005JA011430. [Link]

Eselevich, V. G. and M. A. Filippov, 1988: An investigation of the heliospheric current sheet (HCS) structure. Planet. Space Sci., 36, 105-115, doi: 10.1016/00320633(88)90046-3. [Link]

Fry, C. D., W. Sun, C. S. Deehr, M. Dryer, Z. Smith, S.I. Akasofu, M. Tokumaru, and M. Kojima, 2001: Improvements to the HAF solar wind model for space weather predictions. J. Geophys. Res., 106, 2098521001, doi: 10.1029/2000JA000220. [Link]

Gosling, J. T., G. Borrini, J. R. Asbridge, S. J. Bame, W. C. Feldman, and R. T. Hansen, 1981: Coronal streamers in the solar wind at 1 AU. J. Geophys. Res., 86, 54385448, doi: 10.1029/JA086iA07p05438. [Link]

Hakamada, K. and S.-I. Akasofu, 1982: Simulation of three-dimensional solar wind disturbances and resulting geomagnetic storms. Space Sci.Rev., 31, 3-70, doi: 10.1007/BF00349000. [Link]

Han, S. M., 1977: A numerical study of two dimensional time-dependent magnetohydrodynamic flows. Ph.D. Thesis, University of Alabama in Huntsville, Alabama, USA.

Han, S. M., S. T. Wu, and M. Dryer, 1988: A three-dimensional, time-dependent numerical modeling of supersonic, super-Alfvénic MHD flow. Comput. Fluids, 16, 81-103, doi: 10.1016/0045-7930(88)90040-0. [Link]

Hu, Y. Q., X. S. Feng, S. T. Wu, and W. B. Song, 2008: Three-dimensional MHD modeling of the global corona throughout solar cycle 23. J. Geophys. Res., 113, doi: 10.1029/2007JA012750. [Link]

Lax, P. and B. Wendroff, 1960: Systems of conservation laws. Commun. Pure Appl. Math., 13, 217-237, doi: 10.1002/cpa.3160130205. [Link]

Luhmann, J. G., C. O. Lee, Y. Li, C. N. Arge, A. B. Galvin, K. Simunac, C. T. Russell, R. A. Howard, and G. Petrie, 2009: Solar wind sources in the late declining phase of cycle 23: Effects of the weak solar polar field on high speed streams. Sol. Phys., 256, 285-305, doi: 10.1007/s11207-009-9354-5. [Link]

McComas, D. J., R. W. Ebert, H. A. Elliott, B. E. Goldstein, J. T. Gosling, N. A. Schwadron, and R. M. Skoug,
2008: Weaker solar wind from the polar coronal holes and the whole Sun. Geophys. Res. Lett., 35, L18103, doi: 10.1029/2008GL034896. [Link]

Wang, Y. M. and N. R. Sheeley, Jr., 1990: Solar wind speed and coronal flux-tube expansion. Astrophys. J., 355, 726-732, doi: 10.1086/168805. [Link]

Wang, Y. M. and N. R. Sheeley, Jr., 2009: Understanding the geomagnetic precursor of the solar cycle. Astrophys. J., 694, L11-L15, doi: 10.1088/0004-637X/694/1/L11. [Link]

Wang, Y. M., N. R. Sheeley, Jr., and A. G. Nash, 1990: Latitudinal distribution of solar-wind speed from magnetic observations of the Sun. Nature, 347, 439-444, doi: 10.1038/347439a0. [Link]

Wang, Y. M., N. R. Sheeley, Jr., D. G. Socker, R. A. Howard, and N. B. Rich, 2000: The dynamical nature of coronal streamers. J. Geophys. Res., 105, 25133-25142, doi: 10.1029/2000JA000149. [Link]

Wang, Y. M., E. Robbrecht, and N. R. Sheeley, Jr., 2009: On the weakening of the polar magnetic fields during solar cycle 23. Astrophys. J., 707, 1372-1386, doi: 10.1088/0004-637X/707/2/1372. [Link]

Wilcox, J. M. and N. F. Ness, 1965: Quasi-stationary corotating structure in the interplanetary medium. J. Geophys. Res., 70, 5793-5805, doi: 10.1029/JZ070i023p05793. [Link]

Wu,C.C., C.D. Fry, S. T. Wu, M. Dryer, and K. Liou, 2007a: Three-dimensional global simulation of interplanetary coronal mass ejection propagation from the Sun to the heliosphere: Solar event of 12 May 1997. J. Geophys. Res., 112, A09104, doi: 10.1029/2006JA012211. [Link]

Wu, C. C., C. D. Fry, M. Dryer, S. T. Wu, B. Thompson, K. Liou, and X. S. Feng, 2007b: Three-dimensional global simulation of multiple ICMEs' interaction and propagation from the Sun to the heliosphere following the 25-28 October 2003 solar events. Adv. Space Res., 40, 1827-1834, doi: 10.1016/j.asr.2007.06.025. [Link]

Yang, L., X. Feng, C. Xiang, S. Zhang, and S. T. Wu, 2011: Simulation of the unusual solar minimum with 3D SIPCESE MHD model by comparison with multi-satellite observations. Sol. Phys., 271, 91-110, doi: 10.1007/s11 207-011-9785-7. [Link] 